\title{
Beiträge zur Geschichte der Dritten Zwischenzeit
}

\author{
Karl Jansen-Winkeln
}

Ägyptologisches Seminar Freie Universität Berlin

jawinkel@zedat.fu-berlin.de

\section{Abstract}

In this short Beiträge three points relevant to the history of the Third Intermediate Period are presented.

1. The genealogical data of the family of the army scribe Nespaqashuty written on a fragmentary block statue from Karnak have hitherto been misunderstood. The owner of the statue is not Nespaqashuty II, who lived in the time of Siamun, but a grandson of Amenemone I. The statue may have been dedicated by his son Ankhefenkhons during the time of Osorkon II.

2. According to stela Cairo JE 66285 , the Libyan chief and later king Shoshenq I had a statue of his father Nimlot A erected in the temple of Abydos. The offering established for this statue is written with a hieroglyph simply to be read htp "offering," not hntj "statue" or qnyt "portable image" as proposed before.

3. Some aspects of the chronological and political relations between Bocchoris and Shabako and their predecessors Tefnakhte and Piankhy are considered as well as the supposed reason for the attack on Bocchoris by "Sabakôn."

\section{Keywords}

Genealogy Nespaqashuty - stela Cairo JE 66285 - Shabako/Shebitku - Bocchoris Piankhy - Tefnakhte

In der 21. und der ersten Hälfte der 22. Dynastie gab es in Theben eine prominente Familie, deren männliche Mitglieder im Hauptberuf „Armeeschreiber“ $\left(z \check{s} m s^{\varsigma}\right)$ waren. Eine Quelle von besonderer Bedeutung für diese Familie ist das 
Fragment eines Würfelhockers, das Claude Traunecker 1971 veröffentlicht hat. ${ }^{1}$ Die Statue ist schwer beschädigt, die Vorderseite und die linke Seite fehlen vollständig. Die Rückseite war mit sieben Kolumnen einer Totenopferformel beschriftet, möglicherweise gefolgt von einigen biographischen Sätzen (in der 1. Person); nur von den ersten fünf sind noch Reste erhalten, aus denen sich aber kein Zusammenhang gewinnen lässt. Auf der rechten Seite sind Reste von 14 Zeilen erhalten, die trotz starker Lückenhaftigkeit wertvolle Informationen über die Familie enthalten. Auch diese Inschrift beginnt mit einer htp-dj-nswtFormel; der Gott soll „110 Jahren auf Erden“ gewähren, „indem ich seinem Ka diene..... Schon in der 2. Zeile beginnen dann die genealogischen Angaben: $[\ldots]$ mwt [.f] wrt hnrt $n$ Jmn hr $z^{3} 3-n w$ špst ${ }^{2}[\ldots]$. Die Zeilen 3 und 4 sind vollständig zerstört, aber ab Z.5 ist ein längerer Ausschnitt eines Stammbaums erhalten, der sich in Teilen auch auf anderen Denkmälern dieser Familie findet, v.a. auf den beiden Säulenbasen aus Karnak, die von $\underline{D} d-\underline{D} h \mathbf{w} t j-j w . f_{-}{ }^{-} n h(\mathrm{~A})$ beschriftet worden sind. ${ }^{3}$ Die Gemeinsamkeit betrifft eine Folge von Personen namens B3k-n-Hnzw, Sohn des Ns-p3-q3-šwtj, Sohn des P3j-sn, die beiden letzteren mit den Titeln zš nswt $n$ t3 $r \underline{d}$ r.f $m r m \check{s}^{\iota} N N n$ t3 st Mrj-Dhwwtj. Bei B3k$n$-Hnzw sind diese Titel nicht mehr erhalten. Auf den Säulenbasen endet der Stammbaum mit $P 3 j$-sn als entferntestem Vorfahr (dem Urururgroßvater des

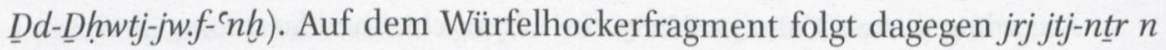

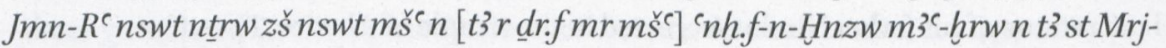

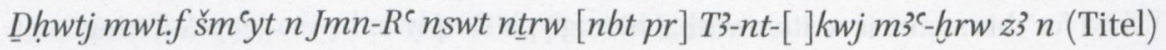
$J j-m-h t p m 3^{c}-h r w z 3$ (Titel) Hr-nht $m 3^{3}-h r w z 3$ [...]. Die Genealogie geht noch weiter, aber sie ist nun extrem lückenhaft. Es scheint sich um Priester des Ptah zu handeln, es ist aber nur noch ein Name Mrj-nfr erhalten.

Traunecker hatte diese Namensfolge so verstanden, dass ' $n h . f-n-H n z w$ der Vater des P3j-sn sei und die Frau T3-n(t)-[ ] kwj seine Mutter, während die folgenden (Männer)Namen wiederum Vater und Großvater (usw.) des ' $n h . f-n$-Hnzw bezeichneten. Auf diese Weise ergäbe sich eine genealogische Kette, die noch $4[+x]$ Generationen über P3j-sn hinausging und somit bis in die 20. oder sogar 19. Dynastie hinaufreichte. Als Statuenbesitzer vermutete er $N s-p 3-q 3-s ̌ w t j$ II,

1 Traunecker, "Un document inédit“.

2 Zweifellos der Titel špst: Er wird in dieser Zeit sehr häufig mit der sitzenden Frau $+t j$ geschrieben, s. Jansen-Winkeln, Spätmittelägyptische Grammatik, § 171, aber das tj kann auch vorangestellt werden s. Habachi, "Clearance of the Area“, 456; Gardiner, "The Hieroglyph with the Value šps“, no. Es handelt sich also nicht um einen Namen $T j$, wie Traunecker annahm, der Name der Frau muss in der Lücke dahinter gefolgt sein.

3 Varille, „Deux bases de Djedthotefankh“; Jansen-Winkeln, Inschriften der Spätzeit II, 92 (17.13). 
dessen Name dann in Z.4 oder 5 zu erwarten wäre, und die in Z.2 erwähnte Frau wäre dann seine Mutter und die Ehefrau des B3k-n-Hnzw (I). ${ }^{4}$

Er hat daher den Stammbaum folgendermaßen dargestellt (Abbt. 1): ${ }^{5}$

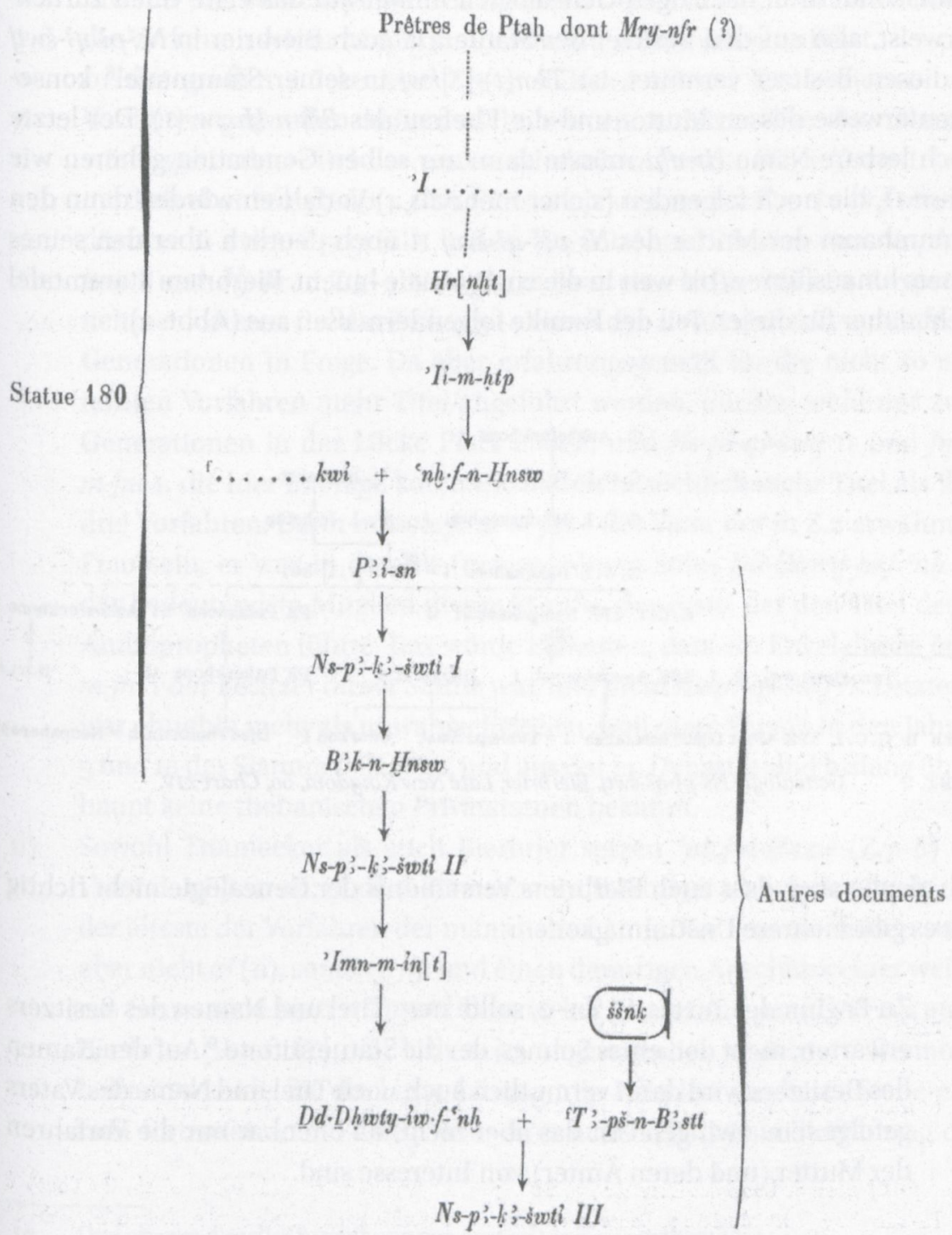

Aввт. 1 Genealogie Ns-p3-q3-šwtj. Traunecker, „Un document inédit", 237.

4 Traunecker, „Un document inédit“, 233, mit n.3.

5 Man beachte, dass in dieser Genealogie der Namen der Mutter $\left({ }_{n} \ldots . . k w i^{i}\right)$ des ${ }^{\circledR} n h . f-n-H n z w$ (vgl. Traunecker, „Un document inédit“, 227 [14]) versehentlich neben 'nh.f-n-Hnzw statt neben Jj-m-htp steht. 
Diese Rekonstruktion der Genealogie ist schon von Morris Bierbrier in gewissen Punkten korrigiert worden: ${ }^{6}$ Er hat zurecht darauf hingewiesen, dass die Angabe "seine Mutter ist die Sängerin des Amun-Re T3-n(t)-[ ]kwj“ in Z.8 sich nicht auf die unmittelbar vorhergehende Person (also $\left.{ }^{6} n h . f-n-H n z w\right)^{7}$ beziehen kann, sondern in derartigen Genealogien immer auf das erste Glied zurückverweist, also auf den Besitzer der Statue. Da auch Bierbrier in Ns-p3-q3-šwtj II diesen Besitzer vermutet, ist $T 3-n(t)$-[ ] $k w j$ in seiner Stammtafel ${ }^{8}$ konsequenterweise dessen Mutter und die Ehefrau des B3k-n-Hnzw (I). Der letzte noch lesbare Name $H r$-nht müsste dann zur selben Generation gehören wie P3j-sn II, die noch folgenden (sicher mehr als 4) Vorfahren würden dann den Stammbaum der Mutter des $N s-p 3 \zeta ̌$ - $q 3$-šwtj II noch deutlich über den seines Vaters hinausführen, bis weit in die 20. Dynastie hinein. Bierbriers Stammtafel sieht daher für diesen Teil der Familie folgendermaßen aus (Abbt. 2):

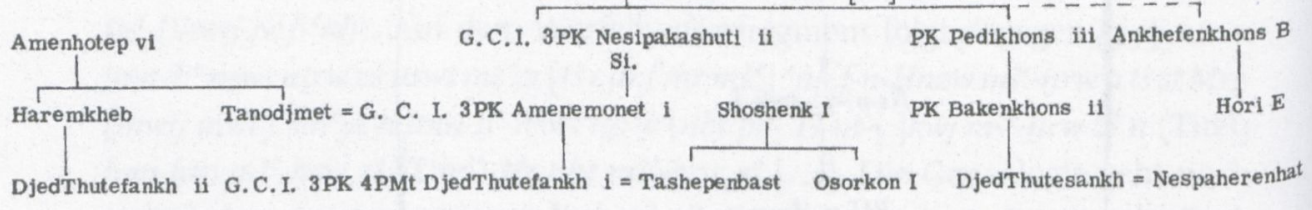

G. Ankhefenkhons A

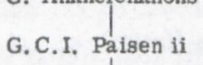

G.C.I. Nesipakashuti i C. I. Imhotep

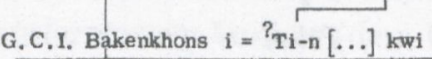
АвВт. 2 Genealogie Ns-p3-q3-šwtj. Bierbrier, Late New Kingdom, 6o, ChartXIV.

Ich glaube aber, dass auch Bierbriers Verständnis der Genealogie nicht richtig ist; es gibt mehrere Unstimmigkeiten:

a) Zu Beginn des Textes, in Z.1-2, sollte man Titel und Namen des Besitzers erwarten, nicht den eines Sohnes, der die Statue stiftete. ${ }^{9}$ Auf den Namen des Besitzers wird dann vermutlich auch noch Titel und Name des Vaters gefolgt sein; zwingend ist das aber nicht, da offenbar nur die Vorfahren der Mutter (und deren Ämter) von Interesse sind.

6 Bierbrier, Late New Kingdom, 6o-62. Bei Payraudeau, Administration, société et pouvoir, 137$3_{8}$ wird der hier interessierende Teil der Familie nicht behandelt.

7 S. oben, Anmerkung 5 .

8 Bierbrier, Late New Kingdom, 60.

9 So Bierbrier, Late New Kingdom, 62. 
b) Die in Z.2 als "seine Mutter" angeführte wrt hnrt nJmn hrr z3 3-nw špst [NN] muß die Mutter des Besitzers sein, und da sie recht hohe Titel hat, sollte sie von einem bedeutenden Vater abstammen. Genau dieselben Titel ${ }^{10}$ führt beispielsweise Hrjt-wbxt, die Tochter des HPA Pajnedjems II. und Enkelin des Mencheperre. ${ }^{11}$ Es ist daher verständlich, dass der Besitzer die Vorfahren seiner Mutter und nicht die seines Vaters anführt.

c) In der großen Lücke von Z.3-5 sollten mehrere Generationen von Vorfahren gestanden haben, nicht nur ein einziger. Die dem Namen vorausgehenden Titel der ersten erhaltenen Person B3k-n-Hnzw I (ver-

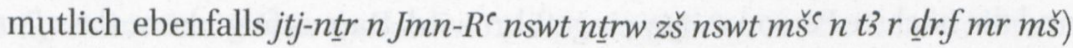
dürften in etwa Z.5 gefüllt haben. Da Titel und Namen von Ns-p3-q3šwtj I und P3j-sn in Z.5-7 eine ganze bzw. eine halbe Zeile in Anspruch nehmen, kämen für die Zeilen 3-4 entweder zwei oder drei zusätzliche Generationen in Frage. Da aber erfahrungsgemäß für die nicht so entfernten Vorfahren mehr Titel angeführt werden, dürften wohl nur zwei Generationen in der Lücke Platz haben, und Ns-p3-q3-šwtj II und Jmn$m$-jnt I, die hier in Frage kommen, hatten tatsächlich mehr Titel als ihre drei Vorfahren. Dann müsste Jmn-m-jnt I der Vater der in Z.2 erwähnten Frau sein, er war in der Tat (neben seinem Sohn $\underline{D} d-\underline{D} h w$ tj-jw. $f$ - $n h$ h A) das bedeutendste Mitglied dieser Familie, der erste, der den Titel des 3 . Amunpropheten führte. Das würde bedeuten, dass ein Enkel dieses Jmn$m$-jnt I der Besitzer dieser Statue war, und nicht $N s-p 3-q 3$-šwtj II. Letzteres war ohnehin mehr als unwahrscheinlich, weil diese Person in den Jahren 5 und 10 des Siamun belegt ist, und aus der 21. Dynastie sind bislang überhaupt keine thebanischen Privatstatuen bekannt.

d) Sowohl Traunecker als auch Bierbrier setzen ' $n$ h.f-n-Hnzw (Z.7-8) als Vater des P3j-sn an; in dem Stammbaum Bierbriers ist ' $n h . f-n-H n z w\left({ }_{n} \mathrm{~A}^{4}\right)$ der älteste der Vorfahren der männlichen Linie. Vor seinem Namen steht aber nicht $z 3(\mathrm{n})$, sondern $j r j$, und einen derartigen Anschluss einer weiteren Generation hatten sowohl Traunecker („engendré par [?]“) ${ }^{12}$ als auch Bierbrier („born [?] of “) ${ }^{13}$ als sehr ungewöhnlich registriert. Dennoch halten es beide für die beste Lösung, die Filiation an dieser Stelle einfach fortzusetzen. ${ }^{14}$ Es liegt aber weit näher, das $j r j$ als Einführung des

10 Und ebenso dieselbe Schreibung von Spst mit vorangestelltem $t j$.

11 Jansen-Winkeln, Inschriften der Spätzeit I, 149-50 (9.42).

12 Traunecker, „Un document inédit“, 223; 226-27 (12).

13 Bierbrier, Late New Kingdom, 61.

14 "The titles of Ankhefenkhons A demonstrate that he must have been an ancestor, doubtless the father, of Paisen“, Bierbrier, Late New Kingdom, $6 \mathbf{1 .}$ 
Stifters der Statue zu interpretieren: "gemacht von“ ‘nh.f-n-Hnzw, der dann vermutlich der Sohn des Statuenbesitzers war. Man würde zwar eigentlich eine Schreibung jrj.n erwarten, aber das gilt für die Einführung des Stifters wie für die Filiation gleichermaßen, $j r j$ ohne $n$ ist bei beiden Interpretationen unüblich.

Wenn aber ${ }^{`} n h . f-n-H n z w$ der Stifter der Statue ist, dann ist $P$ 3j.sn auch hier, wie auf der Säulenbasis aus Karnak, das älteste bekannte Mitglied der Familie. Es wird kein Zufall sein, dass sich die Abfolge ausgerechnet nach P3j-sn ändert und die nächste Person mit jrj eingeführt wird. Daraus folgt dann, dass Jj- $m$ - $h t p$ und $H r-n h t$ Vater und Großvater der Mutter des Stifters (und daher wohl der Frau des Statuenbesitzers) sind, also sehr viel später datieren, als Traunecker und Bierbrier angenommen hatten. Es ergibt sich also folgender Stammbaum:

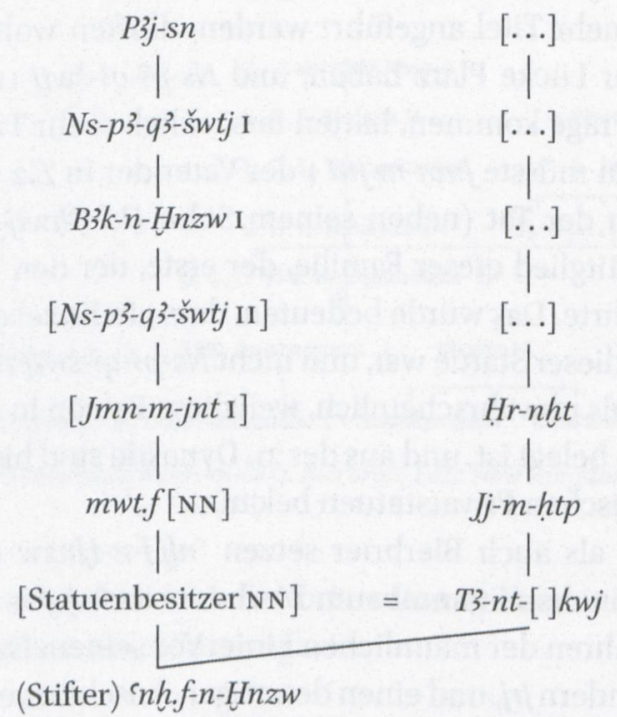

Bemerkenswert ist, dass Jj- $m$ - ht tp, der Vater der T3-n(t)-[ ]kwj und Großvater des mutmaßlichen Stifters der Statue, einige seltene Priestertitel führt, die in derselben Kombination auch bei $N s-p 3$ - $q 3$-šwtj II und $J m n-m-j n t$ I vorkommen:

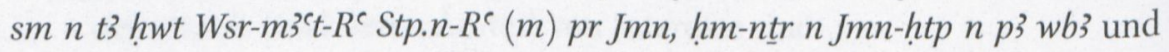
$h m-n t r$ Wsjr W-pqr. Der Vater des Jj-m-htp, Hr-nht, führte diese Titel noch nicht. Also sollte Jj-m-ḥtp sie von der Familie des $\underline{D} d-D h h w t j-j w . f-{ }^{-} n h$ A übernommen haben. Die nachweisbare familiäre Verbindung setzt zwar erst in der nächsten Generation ein: die Tochter des Jj-m-htp heiratet einen Enkel des Jmn$m$-jnt $\mathrm{I}$. Aber es könnte auch schon früher verwandtschaftliche Beziehungen 
zwischen beiden Familien gegeben haben. Allerdings führt $\underline{D} d-D h w t j-j w . f-{ }^{e} n h$ A, der bekannteste Sohn und Amtsnachfolger von Jmn-m-jnt I, gleichfalls die Titel hmm-ntr n Jmn-ḥtp n $p 3$ wb3 und hm-nțr Wsjr W-pqr, und er gehört (sofern der Stammbaum richtig rekonstruiert ist) zur selben Generation wie Jj-m-htp. Aber er könnte diese Ämter später übernommen haben, zumal er vermutlich ein sehr junger Vertreter seiner Generation war ${ }^{15}$ (sein Sohn amtiert noch unter Schoschenk III.).

Wenn dieser Sohn, Ns-p3-q3-šwtj III, diese Titel nicht mehr führt, kann das nichts damit zu tun haben, dass die Familie in Ungnade fiel, denn er selbst ist immerhin Wezir geworden. Dd-Dhwwtj-jw. - $^{-} n h$ h A hatte noch einen gleichnamigen Vetter, dessen Vater und Großvater Hr-3hbjt bzw.Jmn-htp heißen. Dieser Jmn-http (VI) war der Schwiegervater von Jmn-m-jnt I, daher die Verwandtschaft.

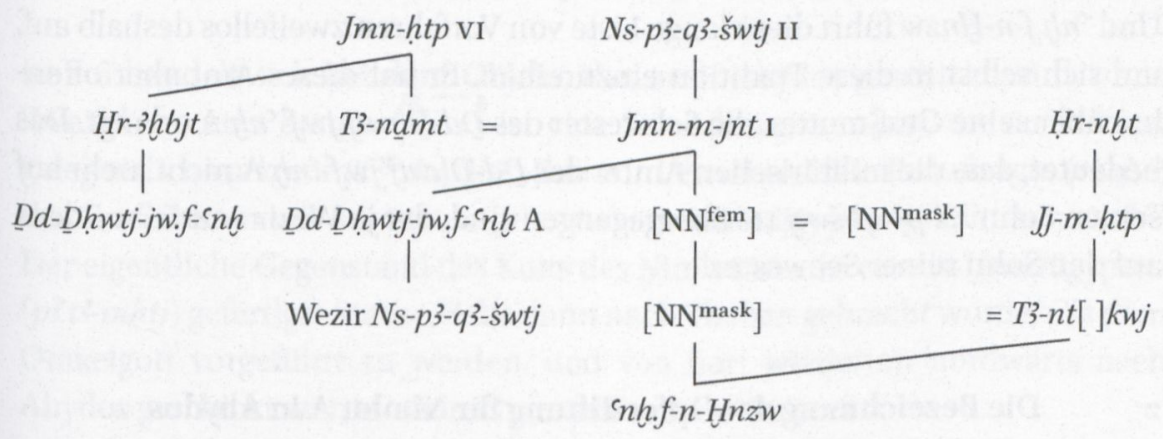

Zwischen den beiden Vettern scheint es zeitweilig eine engere Beziehung gegeben zu haben, denn sie haben gemeinsam eine Statuette der Thoeris ${ }^{16}$ gestiftet. Einer der Gründe für diese recht ungewöhnliche Doppelstiftung dürfte darin liegen, dass die beiden Vettern nicht nur denselben Namen haben, sondern (zumindest auf dieser Statuette) offenbar auch dieselben Titel führen. Der Schutztext der Thoeris für den hm-nțr $n$ Jmn-Re nswt nțrw $m r$ nfrw

${ }_{15}$ Die Ehe mit einer Tochter Schoschenks I. steht dem nicht entgegen, denn diese Tochter könnte ja sehr spät im Leben Schoschenks I. gezeugt worden sein. Es muss auch keineswegs dieser König selbst gewesen sein, der sie $\underline{D} d-\underline{D} h w t j-j w . f-n h$ A zur Frau gab (so Kitchen, Third Intermediate Period, 208). Die Ehe mit einer Prinzessin war sicher auch dann noch eine große Ehre, wenn ihr Vater schon verstorben war.

16 Louvre E.25479, s. zuletzt Jansen-Winkeln, „Vier Denkmäler“, 140-46; Taf.9-11; JansenWinkeln, Inschriften der Spätzeit II, 92-93 (17.14). Vgl. jetzt auch Payraudeau, F. Administration, société et pouvoir, 137 (Fig.33); 612 (279). 
Dd-Dhwtj-jw. $f$ - ${ }^{-} h h$ kann sich also auf beide Personen beziehen. Die Tatsache selbst aber, dass sie gleiche Titel führen, ist sehr bemerkenswert. Denn bei $D d$ -

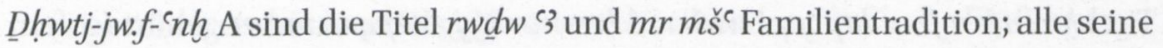
Vorfahren bis hinauf zu P3j-sn führen sie. Dagegen sind Vater und Großvater seines Vetter jeweils nur Amunprophet und Tempelschreiber (zš ḥwt-ntr $n$ pr Jmn), ohne militärische oder andere Titel. Da $D d-D h w t j-j w . f-` n h$ A, wie oben erwähnt, einen Sohn hatte, der noch unter Schoschenk III. amtierte, wäre es leicht möglich, dass er selbst seinem Vater spät im Amt folgte. Die militärischen Ämter des Jmn-m-jnt I könnten daher zunächst seinem Neffen zugefallen und dann erst auf seinen Sohn übergegangen sein.

Der Stifter des von Traunecker publizierten Würfelhockers, 'nh.f-n-Hnzw, trägt übrigens auch den Titel des königlichen Armeeschreibers [des ganzen Landes], sicher der wichtigste Titel dieser Familie, der von $P 3 j$-s $n$ bis zu seinem Urururenkel $\underline{D} d-\underline{D} \not \underline{w} w \mathrm{t} j-j w . f-{ }^{\top} n h$ A jeweils von Vater zu Sohn vererbt worden ist. Und ' $n h . f-n-H n z w$ führt diese lange Liste von Vorfahren zweifellos deshalb auf, um sich selbst in diese Tradition einzureihen. Er hat dieses Amt aber offenbar über seine Großmutter, die Schwester des $\underline{D} d-\underline{D} \not \underline{t} w t j-j w . f-{ }^{`} n h$ A erlangt. Das bedeutet, dass die militärischen Ämter des $\underline{D} d-D h w t j-j w . f-{ }^{`} n h$ A nicht mehr auf seinen Sohn Ns-p $3-q$ - -šwtj III übergegangen sind, der ja Wezir wurde, sondern auf den Sohn seiner Schwester.

Der Großfürst Schoschenk B, der zukünftige König Schoschenk I., hat unter einem seiner Vorgänger, vermutlich Psusennes II., eine Statue seines Vaters Nimlot A "nordwärts" nach Abydos bringen lassen, nachdem zuvor eine Opferstiftung für diese Statue durch Orakel des Amun festgesetzt worden war. Weil der Anfang dieser großen Stele aus Assuangranit, ${ }^{17}$ die auch selbst im Text erwähnt wird (Z.9: ${ }^{c}{ }^{c} w$ m jnr $3 b w$ ), nicht erhalten ist, gibt es einige Unsicherheiten in ihrem Verständnis. ${ }^{18}$ Im folgenden soll es nur um die Bezeichnung dieser Stiftung gehen.

17 Kairo JE 66285, s. Blackman, „The Stela of Shoshenk"; Jansen-Winkeln, Inschriften der Spätzeit I, 159-62 (10.7); Umschrift und Übersetzung auch bei Ritner, The Libyan Anarchy, 166-72.

18 Nicht zuletzt ist es übrigens sonderbar, dass der thebanische Gott Amun eine abydenische Opferstiftung sanktionieren und garantieren soll. 
Der Gegenstand des Orakels wird mehrfach genannt:

- In Z.3 in der Sicherungsformel: der Gott soll alle töten (hldb), die etwas von diesem [Hieroglyphe ungenau] des Osiris NN wegnehmen werden (...ntj jw.w r țj $n k t n p 3 \mathrm{XY} n$ Wsjr $\mathrm{NN}$ )

- In Z.6 geht der Bericht ${ }^{19}$ im Anschluß an das Orakelprotoll weiter: „Dann

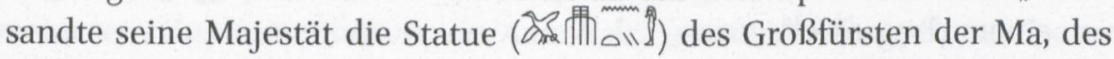
Großen der Großen, Nimlot, gerechtfertigt, nordwärts nach Abydos" (wn.jn

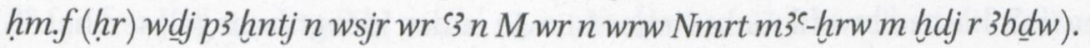

- In Z.9-10 in der Überschrift des eigentlichen Dekrets: „Vorschrift für die Festsetzung des 包 für den Osiris, den Großfürsten der Ma Nimlot“ ( $t p-r d n$ smn $p A$ XY $n$ wsjr wr $3 n$ M Nmrt).

- In Z.25 in der Zusammenfassung: „Das Gottesopfer für das 㛾 des Osiris, des Großfürsten der Ma Nimlot“ ( $p 3$ ḥtp-ntr $n p 3$ XY $n$ wsjr wr $3 n$ MNmrt).

In Z.6 handelt sich bei dem Objekt, das nach Abydos geschickt wird, ohne Zweifel um eine Statue: 鬲ill ist eine Schreibung von hntj, die auch sonst gelegentlich vorkommt. ${ }^{20}$ Auch in Z.10 wird erwähnt, dass Beauftragte ( $\left.r w d w\right)$ des Großfürsten mit der Statue ( $p 3$ twtw) aus Unterägypten gekommen sind. ${ }^{21}$ Der eigentliche Gegenstand des Kults des Nimlot ist also eine in Unterägypten ( $3^{3}$ t3-mhtj) gefertigte Statue, ${ }^{22}$ die dann nach Theben gebracht wurde, um dem Orakelgott vorgeführt zu werden, und von dort wiederum nordwärts nach Abydos geschickt wurde, eine sehr umständliche Prozedur.

In den anderen drei Fällen hatte Aylward M. Blackman zunächst hnt gelesen und an ein Wort für „Altar“ gedacht. ${ }^{23}$ Das war ihm dann von Alan Gardiner ausgeredet worden, der darauf hingewiesen hatte, es wäre merkwürdig, wenn

19 Dessen Beginn auf dem nicht erhaltenen Oberteil der Stele gestanden haben muss.

20 S. Wb. III, $308,5$.

21 Zur Austauschbarkeit von hntj und twtw s. Ockinga, Die Gottebenbildlichkeit, 5-6; 7-8.

22 Die Passage besagt, dass zwei nunmehr zur Opferstiftung gehörige syrische Sklaven aus Unterägypten gebracht wurden "durch die Beauftragten $(r w d w)$ des Großfürsten, die mit der Statue gekommen waren“: das muss ja wohl heißen, daß auch die Statue aus Unterägypten gebracht wurde, vermutlich, weil sie dort (in Memphis?) hergestellt worden war.

23 Blackman, „The Stela of Shoshenk ${ }^{4}, 87$, n.19: „the sign ... is evidently a debased representation of a special form of the hnt-altar". Bei der dafür herangezogenen Parallele (Nelson, „The Decrees of Ramses III“, 232 ff.) sieht das Ideogramm aber deutlich anders aus (ibid., pl. I). 
in Z.6 von einer Statue die Rede sei, in Z.3, 9 und 25 aber von einem Altar. ${ }^{24}$ Blackman hat sich davon überzeugen lassen, und auch andere haben sich dem angeschlossen..$^{25}$ Kenneth Kitchen hat demgegenüber vorgeschlagen, das Ideogramm in Z.3, 9 und 25 qnyt "portable image ${ }^{26}$ zu lesen, und Pascal Vernus versteht es offenbar ebenso („palanquin“). ${ }^{27}$ Es wäre aber schwer einzusehen, wieso man in Z.6 und 10 von einer "Statue" reden sollte, in Z.3, 9 und 25 aber von einem "Tragsessel“.

Statt dessen liegt eine Deutung viel näher, die schon James Henry Breasted ${ }^{28}$ gewählt hatte, obwohl nicht sicher ist, wie er das Ideogramm gelesen hat: ${ }^{29} \mathrm{Er}$ übersetzte in Z.3 und 9 jeweils mit „offering-table“, und das dürfte der Lösung viel näher kommen.

Jan Quaegebeur hat in seiner Untersuchung ${ }^{30}$ des Ausdrucks $p 3$ htp ${ }^{3} w^{c} b$ $n$ Jmn auch die Schreibungen von htp herangezogen, ${ }^{31}$ und dabei zeigt sich, dass diese Schreibungen denen des fraglichen Ideogramms auf der Stele recht ähnlich sein können, in der Dritten Zwischenzeit sogar sehr ähnlich. ${ }^{32}$ Es kann daher kaum zweifelhaft sein, dass es sich bei dem Ideogramm in Z.3, 9 und 25 um ein Zeichen für htp handelt: ein Opfertisch mit einem erhöhten gebogenen Rand an einer Seite und darauf ein Brot. ${ }^{33}$ Wenn man sich die Kontexte ansieht, in denen die Bezeichnungen jeweils vorkommen, ergibt sich auch eindeutig, dass Gardiners Einwand unberechtigt war: in Z. 6 (und 10) ist von der Statue die Rede als dem Objekt des Kults, das an einen anderen Ort gebracht wird (nach Theben bzw. nach Abydos). In Z.3, 9 und 25 geht es dagegen um die Opferstiftung für diese Statue, die nicht beeinträchtigt werden soll (Z.3), die festgesetzt bzw. eingerichtet (smn) wird (Z.9) und deren gesamte Ausstattung zusammengefasst wird (Z.25). In Z.3 ist „etwas vom Opfer wegnehmen“ sicherlich eine wesentlich sinnvollere Formulierung als „etwas von der Statue wegnehmen“, und ebenso

Blackman, „The Stela of Shoshenk“, 94-95.

Z.B. Roeder, Kulte, Orakel und Naturverehrung, 229; 232 ("Abbild“); 236 ("Statue“); Morschauser, Threat-Formulae in Ancient Egypt, 214-15 ("image“); Ritner, The Libyan Anarchy, $166-71$ (hntj „statue“).

Kitchen, Third Intermediate Period, § 239, n.248 (vgl. Wb. V, 52,1-3).

Vernus, „Choix de textes“, 106-07.

Breasted, Ancient Records of Egypt, IV, § 676;679.

Die Wiedergabe bei Mariette, Abydos II, pl.36/37, die Breasteds Übersetzung zugrundeliegt, ist sehr ungenau.

Quaegebeur, „La table d'offrandes“.

Quaegebeur, „La table d'offrandes“, 166-68; 165, Fig. 1-6.

Jansen-Winkeln, Ägyptische Biographien, 201 (12). Auch in der 25./26. Dynastie kann das Zeichen noch so geschrieben werden, vgl. Bothmer, „The Block Statue of Ankh-Khonsu“, Taf.9a, Kol.3, oben (Boston 04.1841).

3 Nicht das hnt-Zeichen (F 63) wie fälschlich bei Blackman. 
ist in Z.9 eine „Vorschrift des Festsetzens (oder Einrichtens) des Opfers“ näherliegend als „eine Vorschrift des Festsetzens der Statue“. In beiden Fällen steht Htp für die Einrichtung des Opfers der Statue. In Z.25 heißt es dmd $p 3$ i htp-ntrr $n$ p' htp $n$ wsjr wr '3 $n$ M Nmrt „Summe / Zusammenfassung des ,Gottesopfers' für das Opfer des Großfürsten der Ma Nimlot". "Gottesopfer“ (hthtp-ntr) ist oft eine Bezeichnung für das (gesamte) Tempelvermögen, ${ }^{34}$ hier wird also konkret gemeint sein:Zusammenfassung des Anteils des (gesamten) Tempelvermögens, das bestimmt ist für das Opfer des $\mathrm{N}$.

Auf jeden Fall wird in Z.3, 9 und 25 nicht das Kultobjekt selbst, die Statue, gemeint sein, sondern das dafür bestimmte Opfer.

Die Eroberung weiter Teile Mittel- und Unterägyptens durch Pianchi ist durch seine große Siegesstele vom Gebel Barkal gut (wenn auch einseitig) dokumentiert, die Eroberung von (zumindest) Memphis durch den ersten König der 25 . Dynastie dagegen nicht $t^{35}$. Es gibt nur einige Hinweise auf den Zusammenstoß mit Bokchoris, die zudem teilweise recht zweifelhaften Wert haben. Der deutlichste findet sich bei Manetho, der in allen erhaltenen Versionen angibt, Sabakôn habe Bokchoris gefangengenommen und lebendig verbrannt.

Das höchste zeitgenössische Datum für die Regierungszeit des Bokchoris ist Jahr $6 \cdot{ }^{36}$ In diesem Jahr ist ein Apisstier bestattet worden, und zu diesem Ereignis gibt es im Serapeum ein Dipinto mit Datum (Jahr 6) und dem Namen des Bokchoris ${ }^{37}$ sowie eine Reihe von (nichtköniglichen) Serapeumstelen aus diesem Jahr. ${ }^{38}$

34 Vgl. Wb. III, 185, 12-13; Meeks, Le grand texte des donations au temple d'Edfou, 55, n.15; Christensen, „Comments on the Stela AEIN 1037“, 15-16; Römer, Gottes- und Priesterherrschaft, § 384-86; Haring, Divine Households, 200.

35 Kürzlich hat Bányai, „Ein Vorschlag zur Chronologie“, die These aufgestellt, Schebitku sei der Vorgänger von Schabako gewesen. In diesem Fall müssten Memphis (und Unterägypten) durch Schebitku (wieder)erobert worden sein. Zu dieser These sind Argumente pro und contra im Mai 2014 auf einem Kolloquium in Münster erörtert worden, dessen Ergebnisse später erscheinen werden.

36 Manetho (nach Africanus) gibt Bokchoris 6 Regierungsjahre; die 44 Jahre nach Eusebius sind in jedem Fall unzutreffend: die Version nach Eusebius gibt für die 23., 24., und 25. Dynastie jeweils 44 Jahre Gesamtlänge.

37 Mariette, Le Sérapéum de Memphis (1857), 24; pl.34; Jansen-Winkeln, Inschriften der Spätzeit II, 375 (41.1).

38 Malinine, u.a., Catalogue des stèles du Sérapéum, Nr.91-102; 117; 121; Jansen-Winkeln, Inschriften der Spätzeit II, 376-81 (41.11-24). Nur die Stele Louvre SN 22 (Malinine, u.a., 
Das früheste zeitgenössische Datum für Schabako ist Jahr 2, belegt auf einer Nilstandsmarke in Theben, ${ }^{39}$ einer Schenkungsstele aus dem Delta (vermutlich aus Pharbaitos $)^{40}$ und auf einer Stele(?) im Serapeum. Diese letztere Stele ist nur durch eine Bemerkung Auguste Mariettes bekannt („une petite stèle grossièrement écrite à l'encre noir “ $)^{41}$ und heute verschollen. In demselben Raum wie sie sei auch "la fin d'une légende royale" gefunden wurde, ${ }^{42}$ eine fragmentarische Kartusche, die auf $1 / u^{\square}$ endet, also vermutlich zu $[D d]$ $k 3 w^{2}-\left[-R^{c}\right.$ (Schabataka) zu ergänzen ist. Jean Vercoutter spricht dagegen nicht von einer Stele, sondern von einer Wandinschrift: „The next date mentioned in the Serapeum is year 2 of Shabaka, which is engraved on the wall of the same chamber in which was buried the Bull which died in year 6 of Bocchoris. ${ }^{43}$ Vercoutters Annahme, die Inschrift aus Jahr 2 des Schabako stamme aus demselben Raum wie die Apisbestattung unter Bokchoris, beruht vermutlich auf dem Plan Mariettes von den Grüften des Serapeums und ihren Bestattungen. ${ }^{44}$ Dort ist der Apis aus Jahr 37 Schoschenks v. alleine in Kammer R begraben, während in Kammer S zwei Apisstiere aus der Zeit des Bokchoris und des Schabako untergebracht sind. Leider sagt Mariette so gut wie nichts über die Begräbnisse selbst, aber seine knappen Bemerkungen (§ 7) zum Apis der 24. Dynastie widersprechen seinem Plan, denn er sagt mehrfach(!), dass er in demselben Raum bestattet wurde wie der im Jahr 37 unter Schoschenk $\{\mathrm{I}\} \mathrm{V}$. begrabene Apis:

Catalogue des stèles du Sérapéum, 83-84, Nr.102) gibt den Tag an: Jahr 6, 1. 3ht, Tag 5 . Aus welcher Quelle Vercoutters Information („Une épitaphe royale“, 341,E; vgl. auch Vercoutter, „The Napatan King and Apis Worship“, 65 und Kitchen, Third Intermediate Period, § 126 [III]; Table 20) stammt, der Apis sei im Jahr 5, 2. šmw, Tag 29 gestorben, ist mir nicht bekannt. Vermutlich handelt es sich nur um eine Rückberechnung, indem man vom Bestattungsdatum im Jahr 6 die üblichen 70 Tage Balsamierungszeit abzieht.

39 Von Beckerath, „The Nile Level Records at Karnak“, 47; 48 (30);Jansen-Winkeln, Inschriften der Spätzeit III, 4 (46.11).

40 Jansen-Winkeln, Inschriften der Spätzeit III, 29 (46.72).

41 Mariette, Le Sérapéum de Memphis, 26 = Maspero und Mariette, Le Sérapéum de Memphis, 184 .

Mariette, Le Sérapéum de Memphis, 27 = Maspero und Mariette, Le Sérapéum de Memphis, 184. Über den Schriftträger (Stele oder Wand) sagt Mariette in diesem Falle nichts.

Vercoutter, "The Napatan King and Apis Worship“, 65 (Hervorhebung im Original). Vercoutter verweist allerdings nur auf Maspero und Mariette, Le Sérapéum de Memphis, 184. Hätte er Informationen gehabt, die Mariettes Angaben korrigieren, sollte man annehmen, dass er sie auch expressis verbis erwähnt hätte.

44 Malinine, u.a., Catalogue des stèles du Sérapéum, Falttafel (Plan A) neben Seite XVI; s. dazu Vercoutter, „The Napatan King and Apis Worship“, 66. 
Il est à noter d'ailleurs que l'Apis mort l'an 37 de Scheschonk IV, dernier roi de la XXII ${ }^{\mathrm{e}}$ dynastie, et l'Apis mort l'an 6 de Bocchoris, l'unique roi de la XXIVe, furent ensevelis dans la même chambre, et que l'étude de la tombe prouve que ces deux Apis occupèrent successivement et sans intermédiaire l'étable sacrée de Memphis. ${ }^{45}$

An einer anderen Stelle wird eine Stele, auf der der Name des Djoser erscheint, ${ }^{46}$ erwähnt als "une petite stèle découverte à la porte même de la chambre sur les parois de laquelle les légendes de Bocchoris et de Scheschonk IV ont été tracées". 47

Dennoch wird man auch diesen Angaben, die sich scheinbar gegenseitig bestätigen, nicht ganz trauen können, ${ }^{48}$ denn für die Stele mit dem Namen des Djoser werden in den Aufzeichnungen Mariettes drei verschiedene Fundorte angegeben, ${ }^{49}$ und von einer Wandinschrift oder einem Dipinto Schoschenks v. im Serapeum scheint sonst nichts bekannt zu sein.

Es bleibt also fraglich, ob und inwieweit Bokchoris und Schabako durch ein gemeinsames Apisbegräbnis verbunden sind ${ }^{50}$ (wodurch natürlich die traditionelle Reihenfolge Schabako-Schebitku bewiesen würde). Bis auf weiteres bleiben Mariettes Angaben widersprüchlich und daher ohne Beweiskraft. Falls Schabako der Vorgänger von Schebitku war, müßte er Memphis spätestens in seinem zweiten Regierungsjahr erobert haben, da es eine Schenkungsstele aus Unterägypten gibt, die in sein Jahr 2 datiert ist. ${ }^{51}$ Auch von Schebitku gibt es eine Schenkungsstele aus Unterägypten, ${ }^{52}$ aber ohne Datum: War er der Eroberer, bleibt das Jahr ungewiss.

Auf jeden Fall ist die Vorgeschichte dieser Eroberung im Gegensatz zum Feldzug des Pianchi nicht bekannt, sie könnte indes durchaus ähnlich gewesen sein:

Vor Pianchis Vorstoß nach Norden hatte Tefnachte einen beträchtlichen Teil des Delta einschließlich Memphis in Besitz genommen, er war der

45 Mariette, Le Sérapéum de Memphis, 24 = Maspero und Mariette, Le Sérapéum de Memphis, $175^{-76 .}$

46 Louvre IM 3036 = Malinine, u.a., Catalogue des stèles du Sérapéum, 93-94, Nr.117.

47 Maspero und Mariette, Le Sérapéum de Memphis, 181.

48 Es ist auch auffällig, dass Gruft R, die nach diesen Aussagen zwei Stierbegräbnisse enthalten haben soll, dem Plan nach die kleinste zu sein scheint.

49 Malinine, u.a., Catalogue des stèles du Sérapéum, 93.

50 Vgl. auch Pope, The Double Kingdom, 262-63.

51 Louvre E.10571, s. Jansen-Winkeln, Inschriften der Spätzeit III, 29 (48.72).

52 New York MMA 65.45, s. Jansen-Winkeln, Inschriften der Spätzeit III, 53 (47.21). 
mächtigste unterägyptische Fürst, und die anderen Kleinkönige und sonstigen Lokalherrscher wurden seine Gefolgsleute. Mit ihnen greift er weiter nach Süden aus und belagert Herakleopolis. Das ist für Pianchi der Moment einzugreifen, zumal auch Nimlot D, der König von Hermopolis, sich Tefnachte anschließt. Der Armee Pianchis gelingt es schließlich, Memphis zu erstürmen, und daraufhin unterwerfen sich ihm nahezu alle Lokalherrscher persönlich, mit Ausnahme von Tefnachte, der nur eine Art Entschuldigungsschreiben schickt und dann vor Abgesandten Pianchis schwört, ihm in Zukunft zu gehorchen. Danach kehrt der nubische König mit der Beute nach Oberägypten zurück.

Früher hatte man fast allgemein angenommen, dass Tefnachte danach den Königstitel annahm, ${ }^{53}$ da es zwei Stelen eines Königs Tefnachte gibt. ${ }^{54}$ Diese Stelen werden jetzt z.T. einem Tefnachte II. (= Manethos Stephinates), vermutlich dem Enkel des Gegners des Pianchi, zugeschrieben, ${ }^{55}$ aber das bleibt unsicher ${ }^{56}$ Aber auch abgesehen von diesen beiden Stelen ist es recht wahrscheinlich, dass sich schon der ältere Tefnachte („I.“) zum König gemacht hatte: Diodor (I.45) bezeichnet ihn als König, und auch seine überragende Stellung im Delta, bedeutender als die der lokalen Könige dort, legt es nahe, dass er irgendwann nach dem Tod Schoschenks v. den Königstitel annahm. In jedem Fall aber muss ihn spätestens sein Sohn Bokchoris angenommen haben, der sicher als König bezeugt ist.

Das Merkwürdige ist nun, dass auch nach dem Feldzug des Pianchi der Machtbereich des Tefnachte und bald danach auch der seines Sohnes kaum beeinträchtigt erscheint. Beide sind nach wie vor Herren des „Grand Royaume d'occident", 57 und zumindest Bokchoris ist wieder im Besitz von Memphis, eine Stadt, die Pianchi erst einige Jahre zuvor erobert hatte. Er ist auch in Tanis im Ostdelta bezeugt, ${ }^{58}$ sein Königtum war also dort anerkannt. Falls Diodors Angabe (1.45) richtig ist, dass ein König Tefnachte („Tnephachthos“), Vater des Bokchoris, einen Feldzug nach Arabien unternahm, müsste auch

Es wäre aber auch nicht ausgeschlossen, dass er dies schon vor dem Feldzug des Pianchi getan hatte, vgl. Jansen-Winkeln, „The Chronology of the Third Intermediate Period“, 262-63.

54 Jansen-Winkeln, Inschriften der Spätzeit II, 372-73 (40.1-2).

55 S. Perdu, „De Stéphinatès à Néchao“, 1221-33, gefolgt von Yoyotte, „Les fondements gèopolitiques", 13-14 und Payraudeau, Administration, société et pouvoir, 23.

56 Kahn, „The Transition from Libyan to Nubian Rule“; vgl. auch Pope, The Double Kingdom, 261-62.

57 Die 24. Dynastie wird bei Manetho ja auch als „saitisch“ gekennzeichnet.

58 Yoyotte, „Notes et documents“, 44-45. 
schon Tefnachte nach dem Rückzug Pianchis wieder im ganzen Delta anerkannt gewesen sein. Das alles ist doch sehr erstaunlich, wenn man den überwältigen Sieg vor Augen hat, den Pianchi wenige Jahre zuvor gegen Tefnachte errungen hatte-jedenfalls seinen eigenen Angaben zufolge. Aber müssen die richtig sein?

Das Bild, das Pianchi auf seiner großen Siegesstele vom Ägypten dieser Zeit entwirft, ist allgemein akzeptiert worden, und es wird ja tatsächlich in vielen Einzelheiten bestätigt; mehrere der dort vorkommenden Personen sind auch aus anderen Quellen bekannt, und sie stimmen in Bezug auf ihre Titel und Herrschaftsräume weitgehend überein. ${ }^{59}$ Es ist allerdings weniger sicher, ob Pianchis Schilderung seines eigenen Erfolges auch so zuverlässig ist. Zum Vergleich: Die Traumstele des Tanutamun berichtet die Wiedereroberung von Memphis und die anschließende Huldigung der Deltafürsten, die Tanutamun ihre Gaben bringen. Die Ähnlichkeit mit dem Ende der Pianchistele ist unübersehbar (und vermutlich auch beabsichtigt). Bei Tanutamun wissen wir allerdings aus den assyrischen Quellen, dass sein Feldzug kein Sieg war, sondern als verheerende Niederlage endete, so dass der Feind sogar Theben erobern und plündern konnte. ${ }^{60}$ Das war bei Pianchi gewiss nicht so, aber auch in seinem Bericht gibt es auffällige Punkte: Der Hauptgegner Tefnachte wird nicht wirklich besiegt und unterwirft sich auch nicht persönlich, und an seiner überragenden Stellung im Delta scheint sich nichts geändert zu haben. Wäre es also nicht denkbar, dass Pianchis Feldzug mit einer Niederlage oder zumindest mit einem Defensiverfolg des Tefnachte endete, vielleicht sogar mit der Wiedergewinnung von Memphis? ${ }^{61}$ Auffällig ist ja auch, dass Pianchi (außer in Napata) seinen Sieg nicht durch neue Denkmäler oder Bauten feiert. In Theben gibt es außer den „Pianchi-Blöcken“ keinerlei Zeugnisse von ihm. Auf einer Stele in Napata, die dort ohnehin niemand zu lesen in der Lage war, konnte man dem lokalen Amun ja eine schöne Geschichte erzählen. Diese Zweifel an Pianchis Darstellung sind selbstverständlich rein spekulativ; immerhin sollte das Beispiel des Tanutamun uns doch davor warnen, sie in allen Punkten für historisch zutreffend zu halten.

59 Vgl. Moje, Herrschaftsräume und Herrschaftswissen, 127-34.

6o Es ist mehr als unwahrscheinlich, dass die Traumstele in der kurzen Zeit zwischen seinem Deltafeldzug und dem Gegenangriff der Assyrer (vgl. Kahn, „The Assyrian Invasions of Egypt“, 262-65) verfasst und hergestellt wurde. Die Niederlage wird schlicht verschwiegen.

61 Memphis ist in jedem Fall noch zu Lebzeiten Pianchis wieder im Machtbereich der 24. Dynastie gewesen, und es nicht recht plausibel, dass Pianchi seinem gefährlichsten Gegner nach einem so überragenden Sieg, wie er ihn schildert, diese Schlüsselstadt und -festung wieder freiwillig überlassen hatte. 
Bei Pianchi war das auslösende Moment für sein Eingreifen der Angriff auf Herakleopolis gewesen, das von Tefnachte und seinen Verbündeten belagert wurde, ${ }^{62}$ jedenfalls wurde er daraufhin von seinen Generälen in Ägypten informiert, es sei Zeit einzugreifen. ${ }^{63}$ Die Gegend von Herakleopolis war seit jeher strategisch wichtig, und gerade in der Dritten Zwischenzeit war es der Grenzbereich zu Oberägypten. Die oberägyptischen Militärbefehlshaber der 21. und 22. Dynastie hatten eine Residenz im nahen El-Hibeh, und noch in der frühen 26. Dynastie war der Flottenkommandant von Herakleopolis auch „Vorsteher von Oberägypten“.

Es gibt nun einen Hinweis darauf, dass auch Bokchoris versuchte, seinen Einfluß bis Herakleopolis auszudehnen oder es sogar schon eingenommen hatte. Auf dem Fragment eines Leintuches (Louvre E.27441) ${ }^{64}$ wird Bokchoris als „[geliebt] von Herischef bezeichnet: $[\cdots]$ 믄

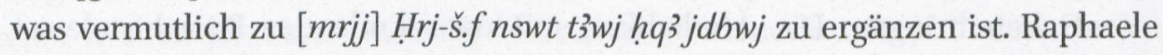
Meffre vermutet, dass dieses Tuch von Bokchoris an Herischef von Herakleopolis gestiftet wurde. In jedem Fall sollte es ein Zeugnis dafür sein, dass Bokchoris seinen Einfluss auch auf Herakleopolis ausgedehnt hatte und er dort als König anerkannt war. ${ }^{65}$

Ein Ausgreifen des Bokchoris nach Herakleopolis dürfte sicher die nubischen Herren Oberägyptens alarmiert haben. Zwar wissen wir nicht, ob es vor der Eroberung des Sabakôn Kämpfe mit Bokchoris gegeben hat, aber das berühmte Gefäß aus Etrurien, ${ }^{66}$ das Bokchoris in Begleitung von Göttern über nubischen Gefangenen zeigt, spricht dafür, dass auch er die Nubier als seine Gegner sah.

Manethos Nachricht, Sabakôn habe Bokchoris lebendig verbrennen lassen, ist angezweifelt worden, ${ }^{67}$ und es wäre in der Tat sehr ungewöhnlich. Zwar gibt es eine Todesstrafe durch Feuer in dieser Zeit, wie die Inschrift des

62 Siegesstele des Pianchi, Z.2-5, vgl. Grimal, La stèle triomphale de Pi('ankh)y, 12-15.

63 Pianchi selbst wird angeblich erst aktiv, als man ihm meldet, dass auch Nimlot von Hermopolis von ihm abgefallen ist und sich Tefnachte angeschlossen hat. Damit konnte ein persönlicher "Verrat“ als Kriegsgrund angeführt werden.

64 Münzen und Medaillen, Werke ägyptischer Kunst, 55-56; Taf.21 (Nr.101); s. jetzt Meffre, D'Héracléopolis à Hermopolis, 150-51 (57).

65 Meffre, D’Héracléopolis à Hermopolis, 363-64; vgl. auch 354; 382.

66 Ridgway, „The Rehabilitation of Bocchoris“, 144 (b); Hölbl, Beziehungen der ägyptischen Kultur zu Altitalien I, 81-94; II, 40-41 (122); Taf.28-30; Farbtafel III.

67 Vgl. etwa Schneider, Lexikon der Pharaonen, 138; Kitchen, Third Intermediate Period, 377 , n.763; dagegen Leahy, „Death by Fire“, 201. 
Hohenpriesters Osorkon B zeigt, ${ }^{68}$ aber die Todesstrafe gegen einen Fürsten oder König würde man in dieser Zeit nicht erwarten. Pianchi hatte die gegnerischen Könige und Fürsten als Unterworfene dargestellt, aber zugleich ihren Rang angegeben und respektiert. Falls Manethos Information richtig ist, müsste es einen besonderen Grund für diese Hinrichtung gegeben haben. Anthony Leahy hat vermutet, der Bruch des Gefolgschaftseids, der auf der Pianchistele behauptet wird, sei dieser Grund. ${ }^{69}$ Allerdings wird auch Nimlot D auf der Pianchistele als ein Gefolgsmann des nubischen Königs dargestellt, der die Seiten gewechselt und damit die Gefolgschaft aufgekündigt hat, ohne dass ihn dieses Verhalten den Kopf kostete. Vielleicht ist der Grund für die besonders rücksichtslose Behandlung des Bokchoris eher darin zu suchen, das er nicht als „legitimer“ König betrachtet wurde. Sein Vater Tefnachte ist ja (allenfalls) gegen Ende seiner Laufbahn König geworden ${ }^{70}$ und wenn er wirklich der Enkel eines Wezirs war, ${ }^{71}$ könnte es gut sein, dass er auch seinen Rang als Großfürst der Libu und der Meschwesch nicht geerbt hatte. Bei solch einem „König“ ohne alle dynastischen Traditionen hätte man vielleicht weniger Hemmungen gehabt ihn hinzurichten. Dafür, dass Bokchoris in der Tat beseitigt wurde, könnte im übrigen eine weitere Angabe bei Manetho sprechen: Falls der „König“ Ammeris („der Äthiopier“), den Manetho (nur nach Eusebius) als ersten Herrscher der (proto-)saitischen 26. Dynastie aufführt, in Wirklichkeit ein nubischer (Militär)Gouverneur war, wie öfter angenommen wird, ${ }^{72}$ würde das ja bedeuten, dass die dynastische Folge unterbrochen war. ${ }^{73}$ Sie wurde dann erst wieder-sehr wahrscheinlich jedenfalls—durch Stephinates (bzw. Tefnachte II.) fortgesetzt. Ein Aspekt der ägyptischen Geschichte dieser Zeit ist also eine Art Zweikampf zwischen der saitischen und der nubischen Herrscherfamilie.

68 Caminos, The Chronicle of Osorkon; § 65; 69 (j) (vgl. zur Stelle auch Vittmann, Der demotische Papyrus Rylands 9, 618). Müller-Wollermann, Vergehen und Strafen, 197 bestreitet, dass es sich beim Feuertod um eine sozusagen „reguläre“ Art der Todesstrafe handelte. Zum Tod durch Verbrennen in Ägypten vgl. auch Holm, „The Fiery Furnace“, 92-100.

69 Leahy, „Death by Fire“, 201.

70 Das heißt, falls Tefnachte (I.) schon König war, hat er sich selbst dazu gemacht, falls er es noch nicht war, hat sich erst Bokchoris den Königstitel zugelegt.

71 Statue Florenz 1777, s. Jansen-Winkeln, Inschriften der Spätzeit II, 270-71 (28.11); vgl. aber auch Yoyotte, „Les fondements géopolitiques", 11 .

72 Vgl. Kitchen, Third Intermediate Period, § 116-18; Schneider, Lexikon der Pharaonen, 105; Perdu, „De Stéphinatès à Néchao“, 1219 .

73 Sofern eine Stele aus dem Jahr 4, auf der Schabako vor Neith und Osiris dargestellt ist, wie anzunehmen aus Sais stammt, wäre das ein weiterer Hinweis auf solch eine Unterbrechung, vgl. Pope, The Double Kingdom, 263. 


\section{Nachträge zu Abschnitt 3}

Im Gegensatz zu meinen Ausführungen auf dem Kolloquium in Münster (s. Fußnote 35) bin ich jetzt der Meinung, dass die (neue) Reihenfolge Schebitku-Schabako in der Tat richtig ist und die Namen dann in der späteren Überlieferung (noch vor Herodot) vertauscht worden sind.

\section{Literaturverzeichnis}

Bányai, M. „Ein Vorschlag zur Chronologie der 25. Dynastie in Ägypten“.JEgH 6 (2013): $46-129$.

Beckerath, J. von. "The Nile Level Records at Karnak and their Importance for the History of the Libyan Period (Dynasties XXII and XXIII)“.JARCE 5 (1966): 43-55.

Bierbrier, M.L. The Late New Kingdom in Egypt (c. $1300 \sim 664$ B.C.). A Genealogical and Chronological Investigation. Warminster: Aris \& Phillips, 1975.

Blackman, A.M. „The Stela of Shoshenk, Great Chief of the Meshwesh“. JEA 27 (1941): 85-95; pl. X-XII.

Bothmer, B. „The Block Statue of Ankh-Khonsu in Boston and Cairo“. MDAIK 37 (1981): 75-83; Taf.7-11.

Breasted, J.H. Ancient Records of Egypt, IV. Chicago: The University of Chicago Press, 1906.

Christensen, Th.F. „Comments on the Stela AEIN 1037 (E 872; A 759) Ny Carlsberg Glyptotek, Copenhagen“. GM 65 (1983): 7-24.

Gardiner, A.H. „The Hieroglyph D with the Value Sps“.JEA 37 (1951): 110

Grimal, N.-C. La stèle triomphale de Pi('ankh)y au Musée du Caire. MIFAO 105. Kairo: IFAO, 1981 .

Habachi, L. „Clearance of the Area to the East of Luxor Temple and Discovery of some Objects". ASAE $5^{1}$ (1951): 447-468.

Haring, B. Divine Households. Administrative and Economic Aspects of the New Kingdom Royal Memorial Temples in Western Thebes. Egyptologische Uitgaven XII. Leiden: Nederlands Instituut voor het Nabije Oosten, 1997.

Hölbl, G. Beziehungen der ägyptischen Kultur zu Altitalien I-II. EPRO 62. Leiden: Brill, 1979 .

Holm, T.L. "The Fiery Furnace in the Book of Daniel and the Ancient Near East".JAOS 128 (2008): 85-104.

Jansen-Winkeln, K. Ägyptische Biographien der 22. und 23. Dynastie. ÄUAT 8. Wiesbaden: Harrassowitz Verlag, 1985 . 
Jansen-Winkeln, K. „The Chronology of the Third Intermediate Period: Dyns. 22-24“. In Ancient Egyptian Chronology, E. Hornung, R. Krauss, und D. Warburton, Hrsg., 234-264. Handbook of Oriental Studies I.83. Leiden: Brill, 2006.

Jansen-Winkeln, K. Inschriften der Spätzeit, I:Die 21. Dynastie. Wiesbaden: Harrassowitz Verlag, 2007.

Jansen-Winkeln, K. Inschriften der Spätzeit, II: Die 22-24. Dynastie. Wiesbaden: Harrassowitz Verlag, 2007.

Jansen-Winkeln, K. Inschriften der Spätzeit, III: Die 25. Dynastie. Wiesbaden: Harrassowitz Verlag, 2009.

Jansen-Winkeln, K. Spätmittelägyptische Grammatikder Texte der 3 . Zwischenzeit. ÄUAT 34. Wiesbaden: Harrassowitz Verlag, 1996.

Jansen-Winkeln, K. „Vier Denkmäler einer thebanischen Offiziersfamilie der 22. Dynastie“. SAK 33 (2005): 125-146; Taf.6-11.

Kahn, D. The Assyrian Invasions of Egypt (673-663 B.C.) and the Final Expulsion of the Kushites“. SAK 34 (2006): 251-267.

Kahn, D. „The Transition from Libyan to Nubian Rule in Egypt: Revisiting the Reign of Tefnakht". In The Libyan Period in Egypt. Historical and Cultural Studies into the 21st-24th Dynasties: Proceedings of a Conference at Leiden University 25-27 October 2007, G.P.F. Broekman, R.J. Demarée, und O.E. Kaper, Hrsg., 139-148. Leuven: Peeters, 2009.

Kitchen, K.A. The Third Intermediate Period in Egypt (noo-65o BC). Warminster: Aris \& Phillips, 1995.

Leahy, A. „Death by Fire in Ancient Egypt“. JESHO 27 (1984): 199-206.

Malinine, M., G. Posener, und J. Vercoutter. Catalogue des stèles du Sérapéum de Memphis. Paris: Imprimerie Nationale, 1968.

Mariette, A. Abydos: description des fouilles exécutées sur l'emplacement de cette ville, II. Paris: Imprimerie Nationale, 1880.

Mariette, A. Le Sérapéum de Memphis, Paris 1857.

Maspero, G. und A. Mariette. Le Sérapéum de Memphis, publié d’après le manuscrit de l'auteur par G. Maspero. Paris: F. Vieweg, 1882.

Meeks, D. Le grand texte des donations au temple d'Edfou. BdE 59. Kairo: IFAO, 1972.

Meffre, R. D'Héracléopolis à Hermopolis. La Moyenne Ėgypte durant la Troisième Période intermédiaire $\left(X X I^{e}-X X I V^{e}\right.$ dynasties). Paris: Presses de L'université Paris-Sorbonne, 2015 .

Moje, J. Herrschaftsräume und Herrschaftswissen altägyptischer Lokalregenten. Topoi: Berlin Studies of the Ancient World 21. Berlin: De Gruyter, 2014.

Morschauser, S. Threat-Formulae in Ancient Egypt. Baltimore, Maryland: Halgo, Inc., 1991. 
Müller-Wollermann, R. Vergehen und Strafen. Zur Sanktionierung abweichenden Verhaltens im alten Ägypten. PdÄ 21. Leiden: Brill, 2004.

Münzen, B. und A.G. Medaillen. Werke ägyptischer Kunst. Auktionskatalog 49, Donnerstag, den 27. Juli 1974.

Nelson, H.H. „The Decrees of Ramses III from Karnak“. JAOS 56 (1936): 232-241.

Ockinga, B. Die Gottebenbildlichkeit im Alten Ägypten und im Alten Testament. ÄUAT 7 . Wiesbaden: Harrassowitz Verlag, 1984.

Payraudeau, F. Administration, société et pouvoir à Thèbes sous la XXII ${ }^{e}$ dynastie bubastite. BdE 160. Le Caire: IFAO, 2014.

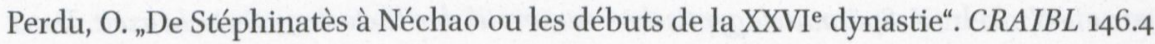
(2002): 1215-1244.

Pope, J. The Double Kingdom under Taharqo. Studies in the History of Kush and Egypt, c. 69o-664 BC. Leiden/Boston: Brill, 2014.

Quaegebeur, J. „La table d'offrandes grande et pure d'Amon“. RdÉ 45 (1994): 155-173.

Ridgway, D. „The Rehabilitation of Bocchoris: Notes and Queries from Italy“. JEA 85 (1999): 143-152.

Ritner, R.K. The Libyan Anarchy. Inscriptions from Egypt's Third Intermediate Period. Atlanta: Society of Biblical Literature, 2009.

Roeder, G. Kulte, Orakel und Naturverehrung im alten Ägypten. Zürich: Artemis-Verlag, 1960.

Römer, M. Gottes- und Priesterherrschaft in Ägypten am Ende des Neuen Reiches. ÄUAT 21. Wiesbaden: Harrassowitz Verlag, 1994.

Schneider, T. Lexikon der Pharaonen. München: Deutscher Taschenbuch Verlag, 1996. Traunecker, $\mathrm{Cl}$. „Un document inédit sur une famille de militaires contemporaine de la XXII ${ }^{e}$ dynastie“. BIFAO 69 (1971): 219-37; pl. XXX-XXXI.

Varille, A. „Deux bases de Djedthotefankh à Karnak“. ASAE $5^{\circ}$ (1950): 249-255.

Vercoutter, J. „Une épitaphe royale inédite du Sérapéum“. MDAIK 16 (1958): 333-345.

Vercoutter, J. „The Napatan King and Apis Worship“. Kush 8 (1960): 62-76.

Vernus, P. "Choix de textes illustrant le temps des rois tanites et libyens". In Tanis. L'or des pharaons, Ausstellungskatalog Paris / Marseille 1987, 102-111. Paris: Association Française d'Action Artistique, 1987.

Vittmann, G. Der demotische Papyrus Rylands 9. ÄUat 38. Wiesbaden: Harrassowitz Verlag 1998.

Yoyotte, J. „Les fondements géopolitiques du pouvoir saïte“. In La XXVI ${ }^{e}$ dynastie continuités et ruptures, D. Devauchelle, Hrsg., 1-32. Paris: Cybèle, 2011.

Yoyotte, J. „Notes et documents pour servir à l'histoire de Tanis“. Kêmi 21 (1971): 35-45. 